Voix et Images

volxetimages

\title{
Automobile - auto - voiture - char : les techniques modernes dans les textes narratifs du Québec des années trente
}

\section{Klaus-Dieter Ertler}

Volume 19, numéro 3 (57), printemps 1994

Science et fiction au Québec : L'émergence d'un savoir

URI : https://id.erudit.org/iderudit/201117ar

DOI : https://doi.org/10.7202/201117ar

Aller au sommaire du numéro

\section{Éditeur(s)}

Université du Québec à Montréal

ISSN

0318-9201 (imprimé)

1705-933X (numérique)

Découvrir la revue

Citer cet article

Ertler, K.-D. (1994). Automobile - auto - voiture - char : les techniques modernes dans les textes narratifs du Québec des années trente. Voix et Images, 19(3), 532-544. https://doi.org/10.7202/201117ar
Résumé de l'article

Résumé

Dans le corpus narratif du Québec de l'époque de la crise, les techniques modernes sont - peut-être contre toute attente - bien représentées. Mais elles ne manquent pas d'y exercer une fonction ambiguë, car les valeurs dominantes de l'époque tendent fréquemment à les stigmatiser, sinon à les exclure. Afin de mieux observer ces mécanismes idéologisants dans quelques textes narratifs des années trente, nous avons mis en valeur les instruments de la Théorie des systèmes (version allemande). Le résultat en est que "la voiture" apparaît comme un facteur catalytique central et diffuse - dans sa fonction de code de communication - toutes sortes de vecteurs stabilisant et/ou subvertissant les discours officiels. 


\title{
Automobile - auto - voiture - char: les techniques modernes dans les textes narratifs du Québec des années trente 1
}

\author{
Klaus-Dieter Ertler, Université de Graz
}

\begin{abstract}
Dans le corpus narratif du Québec de l'époque de la crise, les techniques modernes sont - peut-être contre toute attente - bien représentées. Mais elles ne manquent pas d'y exercer une fonction ambiguë, car les valeurs dominantes de l'époque tendent fréquemment à les stigmatiser, sinon à les exclure. Afin de mieux observer ces mécanismes idéologisants dans quelques textes narratifs. des années trente, nous avons mis en valeur les instruments de la Théorie des systèmes (version allemande). Le résultat en est que "la voiture "apparaît comme un facteur catalytique central et diffuse - dans sa fonction de code de communication - toutes sortes de vecteurs stabilisant et/ou subvertissant les discours officiels.
\end{abstract}

Dans nombre de documents ou de textes littéraires qui datent de la période de la crise, les traces des discours officiels demeurent lisibles, de sorte qu'on pourrait avoir l'impression qu'une seule idéologie — face à l'adversaire, à l'Autre - aurait dominé les champs discursifs.

1. Il est important de souligner dès le départ que les différentes littératures nationales n'ont pas observé l'industrialisation et les techniques de la même façon. Si les littératures française et anglaise au $\mathrm{XIX}^{\mathrm{e}}$ siècle ont largement rendu compte des mutations techniques, les créations poétiques de l'Allemagne se sont plutôt retranchées dans des espaces idylliques éloignés de la modernité (Defizitthese). Par contre, c'est récemment que la critique littéraire s'est intéressée à la question. Voir Harro Segeberg, Hrsg., Technik in der Literatur, Frankfurt/Main, Suhrkamp, 1987; Theo Elm et Hans H. Hiebel, Hrsg., Medien und Maschinen. Literatur im technischen Zeitalter, Freiburg, Rombach Verlag, 1991. Dans le cas du Québec, la question est d'autant plus pertinente parce que les techniques modernes n'ont pas été exclues du discours littéraire. Au contraire, même dans une période à tendance anti-moderne comme les années trente, les vecteurs de la technique y ont trouvé leur place. 
Des idéologèmes comme le "retour à la terre", "l'achat chez nous" ou "l'anti-trust" rappellent le nationalisme à connotation autarcique de l'époque. Le corpus des textes littéraires considérés dans l'institution comme significatifs tend à confirmer ce discours.

Le but de notre analyse est de démontrer que cette homogénéité se révèle factice. Notre texte s'appuiera sur les traces discursives que le système technique a laissées dans les textes. L'isotopie à suivre dans le présent travail sera celle des transports modernes, avec les quelques vecteurs qui en résultent: le trait "américain" du mouvement, la sensation de vitesse et d'indépendance, et le besoin de certains personnages de quitter leur structure sociale stable. Les vecteurs composant cette isotopie s'infiltrent dans la carapace dite homogène du discours social de l'époque. La question qui se pose est alors de savoir s'il y a contamination au niveau discursif et, si oui, dans quel sens cela se manifeste: où le moyen de transport prend-il un rôle déterminant dans la narration? comment arrive-t-il à affecter (positivement ou négativement) la substance idéologique du texte narratif?

Le fait de privilégier l'opposition station/circulation par rapport à la différence ville/campagne nous renvoie au concept de "dromologie ", élaboré par Paul Virilio. Selon cet auteur, la vitesse prend une dimension particulière quand elle est liée à la politique et au social. Elle se transformerait ainsi en "dromocratie". L'approche de Virilio est d'autant plus intéressante qu'elle permet de repérer l'implicite de toute conception de mouvement ou de vitesse et, par conséquent, d'en relever les cristallisations idéologisantes ${ }^{2}$.

Virilio se réfère aux années trente, par le biais, d'une part, de l'impact qu'a connu la Volkswagen dans le contexte politique totalitaire de l'Allemagne, et d'autre part, en se référant à l'effet "salutaire" de la production d'automobiles aux États-Unis, durant la crise. De par l'intérêt qu'elle porte à une perception changeante de la réalité, la dromologie rejoint en partie la théorie luhmannienne des systèmes ${ }^{3}$. Une nouvelle manière de voir les communications, une tentative de délaisser l'observation des images habituelles au profit de nouveaux aspects

2. Virilio insiste sur le rapport qui existe entre le fascisme et la dromocratie tout en rappelant le fait qu'en général, les totalitarismes vont de pair avec les dictatures de la motricité. Voir Paul Virilio, Vitesse et Politique, Paris, Galilée, 1977, p. 27, 116.

3. "[...] je forme ou je déforme ces parcelles de vide, ces trouées, ces creux, c'est un jeu de construction que j'élabore sans accessoires, simplement en étant ici ou là.» Paul Virilio, L'Horizon négatif, Paris, Galilée, 1984, p. 20. Niklas Luhmann est un sociologue allemand dont les très nombreuses productions ont été peu traduites en français. Voir par exemple Amour comme passion. De la codification de l'intimité, Paris, Aubier, coll. «Présence et pensée „, 1990. 
des choses, afin de surpasser la propre cécité de l'observateur: telles sont les visées méthodologiques des deux approches.

Dans un grand nombre des textes narratifs du Québec des années trente, la voiture occupe une place non négligeable. Mettant fin au "règne" du train ${ }^{4}$, elle représente l'un des signes clés de la modernité; pour ces raisons, ses fonctions narrative et idéologique doivent être étudiées.

Avant d'entreprendre l'analyse des textes, il apparaît important de rappeler que presque 200000 véhicules à moteur étaient inscrits dans la seule province de Québec en 1937; dans une petite paroisse comme le Saint-Denis décrit par Horace Miner, il en existait cinq, alors qu'il n'y avait que deux téléphones, et que presque aucune ferme ne disposait d'électricité ${ }^{5}$. En comparaison avec celles des États-Unis ou de l'Ontario, la ferme québécoise possédait relativement peu de commodités modernes. En 1931, 60,3\% des fermes ontariennes disposaient d'une automobile contre seulement $18,9 \%$ des fermes québécoises ${ }^{6}$.

Enfin, n'oublions pas le problème lié à la construction routière, qui confrontait deux intérêts: d'un côté celui des travailleurs, les plus touchés par la crise et pour lesquels la construction représentait un espoir de travail, et de l'autre, les paysans, écologistes avant la lettre, qui voulaient empêcher la destruction de leurs terres ${ }^{7}$. Au niveau textuel, nous verrons que la voiture, comme signe, brouillera les frontières idéologiques parfois trop nettement marquées.

\section{Au cap Blomidon ou la double contrainte}

Le texte de Lionel Groulx, publié sous le pseudonyme d'Alonié de Lestres, offre, du point de vue idéologique, une construction apparemment homogène. Les discours agriculturistes et religieux dominent au sein d'une narration où le principal protagoniste, Jean Bérubé, se con-

4. Voir Wolfgang Schivelbusch, Geschichte der Eisenbabnreise. Zur Industrialisierung von Raum und Zeit im 19. Jabrbundert, München, Hanser, 1977. Selon J. Link et S. Reinecke, la substitution du train par la voiture ("véhicule idéal de la bourgeoisie») signifie l'un des événements discursifs les plus importants qui entraîne aussi une modification énorme au sein du système symbolique. Voir *Autofahren ist wie das Leben. Metamorphosen des Autosymbols in der deutschen Literatur *, H. Segeberg (dir.), Technik in der Literatur, Frankfurt/Main, Suhrkamp, 1987, p. 436-482, 446.

5. Voir Horace Miner, St. Denis, a French-Canadian Parish, Chicago, Phoenix Books / University of Chicago, 1967, p. 256.

6. Everett Hughes, Rencontre de deux mondes (Frencb Canada in Transition), Montréal, Éditions Lucien Parizeau, s. t., 1944, p. 29.

7. Voir Horace Miner, op. cit., p. 253. 
sidère, de par ses souches acadiennes, comme le propriétaire logique de terres ancestrales situées en Nouvelle-Écosse: il essaie de reprendre "sa" terre. Elle se trouve aux mains d'un "Anglais", Hugh Finlay, originaire du Connecticut, et symbole de l'étranger, de l'autre, de l'exclu. Celui-ci est pourtant arrivé à s'établir sur la terre convoitée par Jean Bérubé et à s'y plaire.

Dans ce contexte de prime abord terrien, le vecteur par excellence de la technique de l'époque, l'automobile, est surtout associé à celui qui s'opposera le plus directement à Jean Bérubé: Allan, le fils de Hugh Finlay ${ }^{8}$. Considérée comme objet de loisir, l'auto connote les traits négatifs du fils: voyageur, globe-trotter ( $C B$, p. 55$)$, alcoolique, dissolu, nullement attaché à la terre. La voiture est vue sous un tout autre éclairage dès qu'elle sert de moyen de transport aux catholiques désireux de se rendre à la messe (voir $C B$, p. 74) ou au pèlerinage (p. 180) ${ }^{9}$. Ainsi le récit religieux se retrouve-t-il lié au discours technique et l'auto se "convertit" en signe de progrès, puisqu'elle peut même servir pour les loisirs dominicaux: "Des autos bondés [sic] de voyageurs passaient, suivis [sic] d'un petit nuage de poussière blanche; la route prenait l'animation des jours de dimanche quand il fait beau et qu'on va courir au loin" ( $C B$, p. 78). Le discours agriculturiste se voit donc entièrement harmonisé avec la fonction de loisir de la voiture et l'occupation qui en résulte: le tourisme. "Une auto les avait précédés: des touristes, sans doute, en quête de beaux paysages" ( $C B$, p. 180). Progressivement, l'auto s'intègre dans le récit sans entrer en conflit avec les valeurs diffusées par le texte. Elle sert plutôt de vecteur désignant le dénouement du texte: plus Jean s'approprie la voiture, plus il conquiert la terre. Peu à peu, il introduit le progrès technique à la ferme: "Le camion (à cheval) est du type Sloven, à souples ressorts et à larges pneus. Encore une nouveauté introduite par Jean, à Morse Cottage, pour le transport des fruits " $(C B$, p. 111).

L'influence croissante de Jean Bérubé entraîne graduellement la déchéance de l'héritier anglophone, Allan, ce qui correspond à un

8. Sa première apparition est spectaculaire: "Tout à coup, sur la route de Wolfeville, un [sic] auto déboucha, à une vitesse affolante, dans un tourbillon de poussière. La voiture caracolait et ravageait effroyablement les talus de la route. . Lionel Groulx, Au cap Blomidon, Montréal, Librairie Granger Frères, 1932, p. 59. Dorénavant indiqué dans le texte par le sigle $C B$.

9. Paul Virilio va plus loin en rattachant le comportement mobile général aux grands mouvements politiques: "[...]. motocyclistes, automobilistes, conducteurs de familles, qui reproduisent dans leurs petites évasions quotidiennes, l'ordre dromocratique des grandes invasions. " Paul Virilio, L'Horizon négatif, op. cit., p. 154-155. 
renversement de l'habilité technique: ce n'est plus Allan qui maîtrise l'automobile, mais Jean. Celui-ci trouve l'héritier anglophone dans l'herbe, au bord de la route:

Ils [Jean et Paul] descendirent de voiture et s'approchèrent. Un homme, en costume de highlander, nu-tête, proférait des paroles incohérentes et se débattait dans l'herbe, à deux pieds de la clôture qu'assiégeait furieusement un jeune taureau ( $C B$, p. 96).

Même cet épisode où Allan tente d'éliminer le prétendant-intrus met en scène l'automobile. La narration prend le ton d'un roman policier lorsqu'Allan, représentant du mal, s'érige en bandit de grand chemin contre le futur propriétaire qui lui a, symboliquement, ravi sa place "au volant " de la ferme.

De plus, le discours agriculturiste traditionnel est entièrement dénaturé par une narration relevant des paramètres de la modernité. Ainsi, la lutte entre l'ange et le démon est relatée avec une terminologie propre au discours urbain. Et malgré que le langage utilisé soit étranger au contexte rural du fin fond de la Nouvelle-Écosse, la narration intègre ces éléments sans obstacles:

Comme [Jean] s'apprête à changer de vitesse pour gravir l'avenue montante du chalet, tout à coup, une ombre surgit près de lui, sur l'aile gauche de la voiture. Jean, surexcité, fait un faux mouvement qui étouffe le moteur. Il s'apprête à le réamorcer, lorsque, vis-à-vis [sic] la glace de la portière, un poing se tend, armé d'un pistolet, puis, une face ronde s'avance, avec les yeux fous, et paraît chercher les yeux du chauffeur. Ouvrir la porte de l'auto, bondir sur l'assaillant ( $C B$, p. 224-225).

Jean Bérubé prendra le dessus et récupérera la terre au profit de son peuple, les Acadiens, afin de rompre avec les injustices séculaires dont les "Anglais" sont la cause.

Avec ce roman de 1932, nous nous trouvons face à la situation paradoxale d'un discours agriculturiste, national et catholique, qui intègre sans la moindre résistance l'avalanche discursive propre à la modernité, et ce, avant tout, par le biais de l'automobile. Le texte forme ainsi un conglomérat discursif dont les contradictions dépassent les normes enthymématiques de la société québécoise de l'époque.

\section{Dolorès ou la critique de la civilisation}

Le roman d'Harry Bernard, paru également en 1932, s'inscrit par sa thématique et son style dans le courant psychologisant de l'époque; l'épigraphe ainsi que la topologie se réfèrent néanmoins au roman de la terre. Le texte est dédié entre autres "à Claude-Henri Grignon, à Adolphe Nantel, hommes du Nord, bons écrivains" et contient la 
remarque suivante: "ce livre, où j'ai voulu rappeler l'âme et le visage de leur pays ${ }^{10}$."

Bien que la narration puise ses valeurs dans le discours urbain, elle se réfère principalement aux paysages du Nord. L'avocat, Jacques Forest, est censé éclaircir un décès mystérieux loin de la ville, mais le hasard veut qu'il y tombe amoureux de Dolorès. C'est la raison pour laquelle il ne peut plus mener à bien son entreprise. Dolorès - ellemême citadine retirée à la campagne - idéalise trop la terre pour être crédible.

Les transports modernes, en particulier le train et la voiture, deviennent subrepticement des codes de communication qui dirigent l'action. Par ce moyen, une critique de la civilisation prend forme: l'accident, dont le protagoniste sera témoin, jette les bases du développement idéologique dans la narration. Le voyage de Jacques Forest commence mal et son attitude face à la technique et à la modernité s'avère négative.

Évidemment un malheur vient d'arriver. Je m'approche et j'aperçois sur la voie, entre les rails, un corps étendu. L'homme peut avoir cinquante ans. C'est un pauvre diable mal vêtu, à la barbe en broussaille. Il a les yeux ouverts, des yeux fixes, déjà vitreux, et du sang qui coule des tempes sur les joues. On ne s'explique pas l'accident ( $D$, p. 26).

En tant que catalyseur de la civilisation et du progrès, l'automobile en reflète les signes les plus négatifs. Au deuxième chapitre, par le biais d'un réquisitoire contre la société de loisirs, le narrateur construit un texte imprégné d'une idéologie fortement anti-technique. Comme c'était le cas dans le roman de Groulx, l'automobile, les routes et le tourisme ne s'adaptent plus au discours dominant du texte. Même si l'isotopie négative du roman s'ouvre sur l'automobile, elle abonde par la suite en multiples connotations relatives au sens des valeurs diffusées officiellement par les instances politiques et religieuses. L'énumération reste le procédé narratif privilégié afin de mieux rendre compte de la force destructrice de la civilisation:

Hôtelleries, pensions de famille, magasins à devanture criarde. Partout des postes de ravitaillement pour autos [...] des salles de danse éclairées de lumière multicolore [...]. Ce pays est rongé par le tourisme, lèpre de la nature et du paysage [...]. Route d'asphalte. Les autos fuient, bolides sur roues. Hurlements de klaxons, senteurs d'essence et d'huile, grincement d'embrayage mal calculés [...]. Des lacs clapotent, remplis de Juifs en vacances ( $D$, p. 29-30; voir aussi p. 28).

10. Harry Bernard, Dolorès, Montréal, Éditions Albert Lévesque, 1932, p. 7. Dorénavant $D$, indiqué dans le texte. 
L'automobile entraîne la création de cette "insinuante villégiature moderne" $(D$, p. 30), dont le narrateur parle en développant sa thèse. Lorsque Jacques Forest continue son voyage en voiture de location avec chauffeur (Hilarion), les descriptions font partie de l'isotopie du discours anti-technique: "Il faut avoir vu cet insolite monument d'acier martyrisé..." En même temps il y intègre sa propre terminologie: "[...] dès qu'on parle auto, mécanique, cylindres et pistons, il [Hilarion] s'anime. Sa voiture et lui ne font qu'un ${ }^{11}$ " $(D, \mathrm{p}$. 35). Ce sont finalement une panne électrique et le taux alcoolique élevé du chauffeur qui détournent l'avocat montréalais de ses objectifs professionnels.

Quand Jacques s'installe chez Dolorès, qui vit loin de toute civilisation, de toute circulation, il prend conscience de sa dépendance aux facilités techniques. La voiture devient tout à coup le cordon ombilical qui le relie au monde extérieur, au réel, donc à la civilisation. "[...] je me dis que le spectacle de l'auto avec tout ce qu'il comporte [...] est le plus détestable que je connaisse" $(D$, p. 90$)$. Après la vie idyllique en compagnie de Dolorès, Jacques retrouve la ville, mais il ne retrouvera plus jamais le statu quo ante.

Le texte en question est d'autant plus significatif qu'il n'est pas celui d'un roman régionaliste au sein duquel le discours technique ferait incursion. Au contraire, dans l'histoire à forte dominance psychologique et intimiste, le narrateur cherche à exclure toute intrusion de modernité sous forme technique. Mais il se retrouve pris au piège d'une contradiction flagrante, en refusant le mode de vie urbain tout en perpétuant, par son exemple, le comportement citadin: la dépendance aux moyens de transport, la vie en rêve - donc de loisirs - , sa propre présence dans la nature et, en fin de compte, l'infidélité envers sa fiancée montréalaise. Plusieurs valeurs discréditées dans le texté, en particulier celles où la voiture joue un rôle catalyseur, seront contredites par le comportement du narrateur.

Le discours technique modalise le texte, dans la mesure où il est utilisé pour sa propre contradiction : la voiture est, malgré sa connotation négative, omniprésente et indispensable. De la même manière, les multiples interpénétrations entre l'homme et la technique, sous forme d'anthropomorphisme et de technomorphisme, y contribuent: "L'homme ne connaît pas le repos. Il est né pour travailler, à la sueur

11. „Désir d'un corps métallique, le passager enclos dans l'habitacle automobile répète l'accouplement premier. Comme si l'Occident matérialiste, avec la révolution des transports, installait sa métempsycose dans le présent du corps" (Paul Virilio, L'Horizon négatif, op. cit., p. 51). 
de son corps [...]. Il n'est qu'une vis secondaire, un boulon de chair et de sang, jeté dans le rouage complexe de l'humanité" $(D$, p. 87). En critiquant ouvertement l'entreprise de la colonisation, le texte se retrouve encore plus attaché à la terre et à la nature que ne l'est le discours officiel. Ses failles démontrent pourtant sa propre incohérence. Abstraction faite des remarques extrêmement nationalistes et xénophobes, le roman n'est pas sans rappeler certains discours écologiques de notre fin de millénaire.

\section{Le Feu intérieur ou la speedomanie}

Un an plus tard, en 1933, paraît le roman de Rex Desmarchais qui n'a plus rien d'un roman de la terre ou d'une critique du discours technique moderne, à l'exception de quelques souvenirs d'enfance du personnage principal. L'action du récit se déroule en ville, où l'automobile occupe une place importante et s'avère le vecteur de communication par excellence. Elle représente en quelque sorte le centre magnétique du code social, générant et dirigeant les communications entre les personnages. Il n'est donc pas étonnant qu'elle soit présente dès l'incipit :

[...] Le taxi roulait sans bruit sur l'avenue du Parc; en ce moment à la hauteur de la croix lumineuse. Des automobiles, filant vers le sud, les croisaient. Aux lueurs fugitives des phares et des réverbères, la jeune fille entrevoyait la figure de son compagnon ${ }^{12}$.

L'isotopie découlant de la valeur "automobile" est liée au statut économique de son utilisateur. Marthe Vallières, fille d'une grande famille nantie, et Robert Leval, écrivain et instituteur au salaire modeste, représentent respectivement le luxe dont l'auto fait partie et la misère noire. Gisèle rappelle à son amie Marthe ce statut social: "Crois-tu que tu pourrais te passer de toilettes, d'automobile, de villégiature, d'une bonne?" (FI, p. 55). Par conséquent, la valorisation de la voiture implique une série d'idéologèmes, de la même façon que son refus en entraîne d'autres. Rappelons-nous le texte précédemment étudié, où les mêmes facteurs (l'auto, la villégiature et la mode) ont été profondément méprisés.

Il en résulte que le discours technique moderne se trouve lié à un système sémantique où la voiture, par sa fonctionnalité performative, occupe une place importante, combinée à d'autres facteurs d'origine citadine:

12. Rex Desmarchais, Le Feu intérieur, Montréal, Éditions Albert Lévesque, 1933, p. 11. Dorénavant FI, indiqué dans le texte. 
[Philippe, un ami de Marthe] portait un complet beige, une cravate, un chapeau de même nuance. Une montre bracelet, une perle piquée à la cravate, complétaient sa sobre et parfaite tenue de ville. Il ramena les trois jeunes filles à Outremont dans son torpédo [automobile] vert jade, poli comme un bijou (FI, p. 56).

Jules [un autre ami] a tout. Un nom, de l'avenir, de la fortune, une automobile (FI, p. 69).

Le mariage des deux partenaires, inégaux au plan social, agit comme une stratégie narrative qui permet que Robert prenne le volant lors du voyage de noces à la Mecque de la société urbanisée: Atlantic City. À l'occasion d'un arrêt chez sa grand-tante, qui vit à la campagne, Robert affiche son nouveau statut social par le biais de la voiture. Les discours citadin et rural se chevauchent alors, Robert se souvenant de la perception différente de la réalité qu'il avait durant son enfance (voir FI, p. 103). Cette brève percée de l'anti-modernisme représente les derniers échos de la valorisation de la terre. Les cultivateurs, quant à eux, ne cessent d'admirer ce moyen de transport individuel permettant le luxe d'un déplacement rapide: "T'as un ben beau char" "Oui, il a acheté ce torpédo léger. Mais l'an prochain, il aura un Packard "(FI, p. 110).

La frénésie de la vie moderne, représentée par la voiture, donne à la narration une allure vertigineuse. La vitesse effrénée de l'automobile, correspondant à la philosophie d'“aller de plus en plus vite pour n'atteindre aucun but" (FI, p. 119), se traduit par une syntaxe brisée et par l'énumération d'impressions fugaces qui s'opposent à la forme homogène qu'on attribue habituellement à la littérature régionaliste: "Virages, descentes, montées, folles accélérations, brusques freinages" (FI, p. 119). Le transport, vu sous ses côtés ludiques prend ainsi le dessus et se manifeste aussi bien au niveau sémantique de l'isotopie qu'à celui des structures narratives. La "speedomanie", cette "fringale de vertige" ( $F I$, p. 129) pour Marthe, traverse le texte. À la manière d'une drogue, elle neutralise l'orientation et bouleverse les paramètres de l'espace-temps, permettant ainsi aux protagonistes de fuir la réalité, dimension pourtant prépondérante des textes régionalistes de l'époque :

"Plus vite, disait Marthe. La vitesse me fait du bien." [...] Sur l'avenue Maplewood, semblable à une piste, il lança son torpédo. Sensation d'immobilité: les réverbères, les maisons se livraient en sens inverse à une course folle (FI, p. 133).

La voiture joue donc un rôle manifeste dans le roman, non seulement en tant que composante de l'isotopie du luxe, ou comme moyen d'"évasion", mais aussi comme focalisation de la communication. De 
nombreux passages du texte se déroulent dans l'espace réduit et intime de la voiture, présentant des conversations comme celle de l'incipit, entre Marthe et Robert dans le taxi. Il faut noter que l'espace de l'automobile n'est aucunement réservé aux personnages masculins ou aux couples; les femmes aussi y ont droit: "Comme ce soir, elles [Marthe et ses amies] sortent ensemble en automobile" (FI, p. 124).

Le roman, qui se veut psychologisant en mettant en scène une relation de couple morose, diffuse une isotopie de luxe et de loisirs qui ouvre une brèche au discours technique moderne. Ainsi l'automobile, facteur central dans la société de Marthe, se manifeste-t-elle dès l'incipit et aura pour effet d'étouffer toute objection de la part de Robert, dont les perspectives provinciales, rurales, et partant critiques, sont somme toute à peu près absentes du roman.

\section{Dans les ombres ou le catalyseur de la tragédie}

Le roman psychologique d'Eva Sénécal, publié en 1931, développe la double relation sentimentale de Camille: d'un côté, l'élu, Robert L'Heureux, qui est directeur d'une succursale bancaire du Nord; de l'autre, l'Américain Richard Smith acheteur de lots de bois au Québec. Ce qui scinde provisoirement le destin de la jeune femme s'inscrit dans le discours technique moderne: l'accident de voiture. Outre les conséquences qui sont ici plus graves, la même sensation de vitesse et de délire qu'on pouvait repérer dans le texte précédent marque la narration. La densité des énumérations d'événements atteint son apogée au moment crucial de l'accident :

Une fuite joyeuse dans l'automobile qui les [Camille et Robert] emportait vers Québec. [...] un tournant de route, une chute affreuse, des cris, des plaintes, des ténèbres, le cauchemar, une chambre d'hôpital, les religieuses, l'odeur des remèdes et des fleurs [...] Ainsi finissait l'enchantement ${ }^{13}$.

La mauvaise surprise renvoie les deux jeunes gens à leur solitude respective : Robert s'installera dans le Nord et Camille, convalescente, restera chez ses grands-parents.

L'autre amoureux de Camille lui fait vivre des expériences semblables, comme si seuls la vitesse et le danger pouvaient unir les amants. Temps et espace se confondent au plus haut degré, de sorte que l'expression métonymique du progrès, l'automobile, fournit de nouveaux plaisirs liés au vertige. Au point où il y a lieu de se demander ce qui

13. Eva Sénécal, Dans les ombres, Montréal, Éditions Albert Lévesque, 1931, p. 33. Dorénavant $D L O$, indiqué dans le texte. 
reste du refus du discours technique que l'on rattache d'emblée au roman régionaliste.

L'extrait qui suit rend compte des effets déséquilibrants de la vision du monde sous l'impact de la technique moderne:

Richard Smith pressa l'accélérateur de l'automobile. Le paysage se mit à fuir avec une rapidité vertigineuse. C'était délicieux [...] Tout se brouillait. Les coins de verdure se confondaient avec de grandes étendues de sol rude. Les arbres, les jardins, la maison apparaissaient pour disparaître aussitôt ( $D L O$, p. 46).

Au plan narratif, la confusion des repères et des valeurs due à l'ivresse de la speedomanie fait en sorte que les retrouvailles avec l'esthétique de la terre demeureront impossibles. Les multiples tentatives de récupérer le passé n'arrivent pas à réintroduire les idéologèmes de la terre et de la religion.

\section{Sébastien Pierre ou l'américanité}

Jean-Charles Harvey publia son recueil de nouvelles au milieu des années trente. Le récit éponyme présente un champ de bataille discursif où deux pôles s'affrontent: québécité cléricale anti-matérialiste contre américanité moderne amorale. Par conséquent, les moyens de transport modernes, notamment la voiture, y trouvent leur place: dans le premier volet où la technique se trouve intégrée à une isotopie négative, l'automobile symbolise la force destructrice du matérialisme. Avec l'accident de voiture, un noud d'idéologèmes se crée, donnant à lire, en filigrane, le message suivant: le spirituel ne peut guère être atteint par le matériel, le technique ${ }^{14}$. "[...la cousine de Pierre] est morte d'un accident d'automobile. On a rapporté chez ses parents le corps sanglant de la jeune fille. [...] Un œil est sorti de l'orbite, le visage abîmé d'estafilades, le crâne ouvert [...] ${ }^{15}$ n. Au deuxième volet, Sébastien se transforme en "Tony Jackson " et les valeurs se renversent: l'automobile se voit tout à coup déifiée, correspondant ainsi à la mentalité de la métropole nord-américaine. L'automobile y représente le luxe, l'argent et l'aventure sans que l'aspect moral des activités criminelles de la bande de Tony Jackson soit pris en considération. La narration et ses techniques relèvent du roman policier, au sein duquel l'automobile occupe une fonction pratique, conformément au contexte métropoli-

14. On peut observer le même procédé dans d'autres textes de l'époque, par exemple Harry Bernard, Juana, mon aimée, Montréal, Librairie Granger Frères, 1931, p. 73; J. A. Bernier, On vend le bonbeur, Montréal, Librairie de l'A.c.-f., 1931, p. 91.

15. Jean-Charles Harvey, Sébastien Pierre, Montréal, Stanké, 1985 (1935), p. 27. Dorénavant $S P$ indiqué dans le texte. 
tain. Par conséquent, la voiture n'est pas associée à la sphère du plaisir - comme nous l'avons vu, dans d'autres textes québécoịs - mais c'est sa fonction instrumentale qui domine. Elle sert de moyen de transport aux contrebandiers et aux voleurs et représente pour eux un bouclier contre les menaces qui viennent de l'extérieur du groupe. Vue dans cette perspective, la vitesse n'est pas un plaisir en soi, mais plutôt l'instrument principal des activités criminelles:

Une nuit, qu'il transporte, en automobile, de l'alcool de contrebande à destination de New York, il [White Devil] s'aperçoit que les agents le suivent. Il accélère. Les motocycles accélèrent aussi. Course éperdue à travers la campagne. Devil entend des coups de feu ( $S P$, p. 83).

La perception de la voiture change néanmoins, lorsque Tony Jackson - fuyant en voiture vers le Nord - traverse la frontière canadienne: "Bientôt, il vit devant lui les paisibles villages de son pays. Où allait-il? Il ne le savait guère" (SP, p. 99). Seule compte alors la vitesse, la voiture n'ayant plus de fonction utilitaire.

\section{Trente Arpents ou l'irruption de l'automobile}

Publié en 1938 par Ringuet, ce "dernier" roman de la terre intègre la voiture en tant que métonymie de la modernité, mais il le fait d'une façon extrêmement critique. Ainsi l'automobile fait-elle éclater les conceptions de l'espace, du temps et des cycles séculaires de la vie paysanne pour faire place aux valeurs importées des États-Unis. La valorisation de ce nouveau moyen de transport est double: Euchariste Moisan le voit d'un oil critique tandis que les jeunes s'en servent pour leurs loisirs. Le narrateur omniscient en relève le caractère nocif, et l'automobile - pourtant bien plus répandue qu'au début de la décennie - se trouve clouée au pilori de façon explicite.

On était loin des dimanches d'autrefois, des après-midi douces et un peu mornes passées sur la véranda. L'automobile était venue qui avait changé tout cela. Chaque remise en abritait maintenant une: chez les paysans modestes quelque tacot ramassé dans les parcs d'occasion $[\ldots]^{16}$,

Remarquons finalement que le "règne de l'automobile" a entraîné le vieux paysan à terminer ses jours comme gardien dans un garage américain. Il faut noter également que Trente Arpents ne marque pas

16. Ringuet, Trente Arpents, Montréal, Fides, 1970 (1938), p. 255-256.

17. "The number of motor vehicles registered in the Province of Quebec rose only from 197,917 to 298,688 between 1937 and 1947.. Horace Miner, op. cit., p. 256. Traduction: "Le nombre de véhicules motorisés enregistrés dans la province de Québec s'est accru seulement de 197917 à 298 688, entre 1937 et 1947.» 
seulement la fin du roman de la terre, mais également le fléchissement de la première poussée économique de l'automobile au Québec ${ }^{17}$.

\section{Conclusion}

À la suite de notre analyse de quelques textes narratifs du Québec des années trente, nous pouvons constater que l'automobile, en tant que code de communication du discours technique moderne, était bien représentée dans la mentalité collective. Bien qu'après la crise économique, la province francophone ait développé - selon les modèles européens - une réaction intégriste avec un repli antiscientifique et anti-technique, la philosophie de la "mobilité générale" a pourtant triomphé. À la lecture de ces textes narratifs, nous pouvons affirmer que l'impact important de l'automobile dans la société québécoise y change la perception du réel, à l'instar, bien qu'à un moindre degré, du cheval, du carrosse ou du train au cours des siècles. Mouvement, accélération et vitesse ont transformé une vision homogène du monde, axée sur les valeurs locales, en privilégiant une fragmentation plus prononcée. Le même procédé peut être observé au niveau de la construction narrative: la présence de l'automobile en mouvement fait accélerer les séries d'images de la vitesse, aboutissant parfois au paroxysme de l'accident. Fréquemment, la syntaxe se brise et le texte glisse vers une simple agglomération de séries nominales ou verbales.

Mais l'automobile ne détruit pas' seulement la perception homogène du monde extérieur, elle crée aussi de nouveaux espaces intérieurs qui fonctionnent comme des microsphères intimes. La présence de l'automobile dans l'isotopie du discours technique moderne de l'époque est en quelque sorte obligée, vu l'importance du phénomène au plan social. Même connotée négativement, ou carrément refusée, l'automobile renvoie alors au discours anti-technique. 